Copyright (c) 2007 IEEE. This material is presented to ensure timely dissemination of scholarly and technical work. Copyright and all rights therein are retained by authors or by other copyright holders. All persons copying this information are expected to adhere to the terms and constraints invoked by each author's copyright. In most cases, these works may not be reposted without the explicit permission of the copyright holder. 


\title{
Small-Signal Transfer Functions of the Classical Boost Converter Supplied by Ultracapacitor Banks
}

\author{
R.M.A.S. Rajakaruna \\ School of Electrical and Flectronic Engineering \\ Nanyang Technological University \\ 50 Nanyang Avenue, Singapore 679798
}

\begin{abstract}
.4bstract-Lltracapacitor is an emerging device for energ! storage that can replace batteries in some short-term applications. Due to the fact that the terminal voltage of at ultracapacitor varies with the level of energy stored, a power electronic converter with voltage boosting capability is usually needed at its terminals to interface with a load operating at a constant voltage. For the case of a classical boost de-de converter supplying a resistive load by drawing energy from a bank of ultracapacitors, the paper derives small-signal transfer functions by considering a time-sarying steady-state. To reduce the complexity of resulting exact expressions of poles, highly accurate approximate expressions are also derived. Furthermore, it compares how the control characteristics change when a constant voltage source such as a battery replaces the ultracapacitor bank. A numerical example is presented to demonstrate the accuracy of the approximate values of poles and zeros and to describe how the poles and zeros vary with the duty ratio.
\end{abstract}

\section{INTROLULTION}

Ultracapacitor is an electrochemical type capacitor which offers large capacitances in the order of thousands of Farads but at a low rated voltage of about $2.5 \mathrm{~V}[1-3]$. The energy density of an ultracapacitor is about 100 times larger than a conventional electrolytic capacitor and its power density is about 10 times larger than a lead-acid battery. After its introduction to the market with large capacitances in the $1990 \%$ s, it has become attractive for many industrial applications such as hybrid electric vehicles, power quality applications etc. because they are ideally suited to deliver high powers for a short duration of time [4-7]. Ultra-capacitors also have a number of other attractive properties, such as fast charge-discharge capability, longer life, no-maintenance and environmental friendliness due to no harmful emissions.

Despite the above attractive features, they also possess some undesirable features such as the low voltage rating. variable terminal voltage compared to batteries, larger series resistance compared to conventional capacitors, larger leakage current and higher nonlinearity in the capacitance with voltage. [1-3] Due to the low rated voltage, many cells are connected in series to form a bank of capacitors that has sufficiently high rated voltage. In such banks of capacitors. voltage balancing by either active or passive balancing is also necessary to prevent damages of cells due to overvoltage. Furthermore, due to the variation of terminal voltage with the energy stored, a power electronic converter is usually needed at the terminal of a bank of ultracapacitors to convert it to a constant voltage source or a sink that is comparable with a battery. In order to minimize the number of series connected cells in the bank, a voltage boosting converter such as a classical boost converter [4] and flyback converter [7] is usually used in industrial applications.

In the case of a classical boost converter supplied by a constant voltage source such as a battery. some specilic control problems such as the effects of Right Hand Plane (RHP) zero are discussed and some alternative converter have been proposed $[8,9]$. However, in designing a feedback controller for a converter supplied by a bank of ultracapacitors, it is found that there is hardly any literature available to understand the behaviour of the onen-loop system. Therefore, the aim of this paper is to derive a smallsignal model using fundamental concepts so that it can bo used to understand the behaviour of the open-loop system before designing a feedback controller to achieve constant load voltage.

In section Il of the paper, a simple electrical model of the switching circuit is used to derive the average state equation and to identify the characteristics of the steady-state. The small-signal transfer functions, the exact and approximate values of poles and zeros are then derived in section III. In order to demonstrate the accuracy of approximate values and to confirm the expected variations of poles and zeros with duty ratio, a numerical example is presented in section IV Finally, the conclusions are presented in section $\mathrm{V}$.

\section{Averacie Si ate SPACI: Mode:}

For the purpose of describing the terminal voltage vs. current characteristic of an ultracapacitor, many models have been developed in the past [1,10,11]. Despite the varying degree of accuracy of the models, almost all of them are too complex to be used in a small-signal analysis due to increased number of order of resulting differential equations. On the other hand, the objective of the analysis made in this paper is to identify the differences in control characteristics due to the replacement of the constant voltage source by a capacitor. Therefore, the ultracapacitor bank is modeled by an ideal eapacitor in this paper. The effect of the equivalent series resistance (ESR) of the capacitors can be analyzed after studying the basic behaviour of the system. Figure 1 illustrates the electrical circuit diagram of the classical boost converter supplied by the bank of ultracapacitors. The stray resistances 
of all the circuit components have been neglected here to focus on the fundamental behaviour of the system. The converter consists of an input inductor $L$. output filter capacitor $C_{t}$, the switching transistor $\mathrm{Sc}$ and the diode $\mathrm{D}_{i}$.

The transistor $S_{\text {i }}$ switches at a high frequency with a constant period $T$ and a variable duty ratio $d$ adjusted by the control circuit, not shown in Fig. I. At the converter output terminals is the load resistor, $R$.

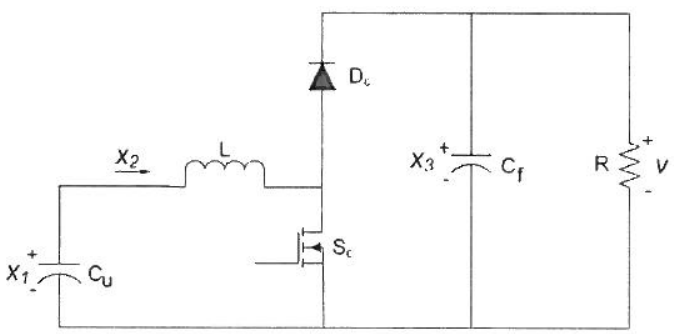

Fig. 1. (ircuit diagram of the classical berst comerter supplied hy a hank of ultratanacitors

\section{A. Arerage Sistem Model}

In the circuit diagram given in Fig. 1, the three states $x_{1}, x_{2}$ and $x_{3}$ are selected as the voltage across the ultracapacitor bank. current through the input inductor and the voltage across the output filter capacitor respectively. The system differential equations for the period $d \cdot T$ during which the transistor is on can be written as.

$\dot{x}_{1}=\left(-1 / C_{11}\right) \dot{x}_{2} \quad \dot{x}_{2}=(1 /$ l. $) x_{1} \quad \dot{x}_{3}=\left(-1 /\left(R C_{1}\right)\right) x_{3}$

which can be expressed in matrix form as $\dot{x}=A_{1} \cdot x$ where.



is the state matrix in the on-state of the transistor and $x=\left(\begin{array}{lll}x_{1} & x_{2} & x_{3}\end{array}\right)^{T}$

is the state vector. Similarly, for the ofl-state of the transistor with the duration $(1-d) T$. system differential equations are given by,

$$
\begin{array}{ll}
\dot{x}_{1}=\left(-1 / C_{u}\right) x_{2} & \dot{x}_{2}=(1 / L)\left(x_{1}-x_{3}\right) \\
\dot{x}_{3}=\left(1 / C_{1}\right)\left(x_{2}-x_{3} / R\right) &
\end{array}
$$

The corresponding state equation can therefore be written as $\dot{x}=A_{2} \cdot x$ where.

$$
A_{2}=\left(\begin{array}{ccc}
0 & -1 / C_{11} & 0 \\
1 / 1 & 0 & -1 / L \\
0 & 1 / C_{1} & -1 /\left(R C_{1}\right)
\end{array}\right)
$$

is the state matrix in the transistor off-state. According to Middlebrook's state space averaging theory [12], the average values of state variables are given by

$$
x=\left[A_{1} \cdot d+A_{2} \cdot(1-d)\right] \cdot x
$$

where the average state matrix $A=A_{1} \cdot d+A_{2} \cdot(1-d)$ can bo expressed in terms of circuit parameters as.

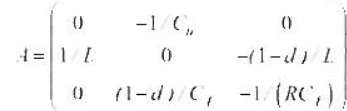

Since the load voltage $r=x_{3}$ is the output variable. the output equation is given by $l=C \cdot x$ where $C=\left(\begin{array}{lll}0 & 0 & 1\end{array}\right)$.

\section{B. Steadi-State Operating Points}

\section{1) DC Steact-State}

Since the derivatives of all the variables are zero at de steadystate, de steady-state relationships can be obtained by $X=A \cdot X=0$ where $X=\left(X_{1}, X, X_{3}\right)^{T}$ is the state vector at steady-state. By taking $D$ as the duty-ratio at the steady-state. following de steady-state equations can be derived.

$X_{2}=0$

$X_{1}=(1-D) \cdot X_{3}$

$X_{3}=(1-I) R \cdot X_{2}$

According to $(8)-(10)$. the only possible steady-state is when all state variables are zero. Since there is no source in the system. initial energy stored in the ultracapacitor bank. filter capacitor and inductor are used to supply the load. Hence. all the variables decay to zero with increasing time. Consequently. the system has no non-zero de steady-state when the converter is in operation. i.e. for any value of $D$. Therelore, for the purpose of designing a controller using conventional methods. a smaltsignal analysis of the system can only be performed by considering a time-varying steady-state.

\section{2). Time-L'aning Stead-State:}

Since the transler functions of interest are from duty-ratio to state variables. the steady-state should be defined as the state of the system when the duty-ratio is constant. With a constant duty-ratio. the variables will change exponentially as demonstrated by computer simulation results in Section IV. Therefore, the state vector in steady-state can be expressed as

$X(t)=\left(\begin{array}{lll}X_{1} & X_{2} & X_{3}\end{array}\right)^{T} \cdot e^{-t t_{1} t}$

where $X_{1}, X_{2}, X_{3}$ are the initial values of state variables and $\omega_{1}$ is the steady-state frequency in $\mathrm{rad} / \mathrm{s}$. Then, substituting (11) in (6). lollowing steady-state relationships can be obtained.

$$
\begin{aligned}
& \omega_{1}=\frac{X_{2}}{C_{n} X_{1}} \\
& -\omega_{1} X_{2}=\frac{1}{L}\left[X_{1}-(1-D) X_{3}\right] \\
& -\omega_{1} X_{3}=\frac{1}{C_{1}}\left[X_{2}(1-D)-\frac{X_{3}}{R}\right]
\end{aligned}
$$

Thus, if two out of five parameters $X_{1}, X_{2}, X_{3}, D$ and $\omega_{1}$ are 
given. the other two parameters can be found using (12)-(14). By taking Laplace translorm of (11). the frequency shift due to time-varying steady-state can be identified.

$$
X(s)=\left(\begin{array}{lll}
x_{1} & X_{2} & x_{3}
\end{array}\right)^{t} \cdot \frac{1}{\left(s+\left(u_{1}\right)\right.}
$$

From (15), it is clear that the eflect of exponentially varying steady-state is only to introduce a frequency shitt by $\omega_{11}$ to the values given by de steady-state. The derivation of transler linetions can be performed as lor a de steady-state. However, the frequency of zeros should be corrected by deducting $\varphi_{\text {, }}$ as they depend on the state vector at steady-state. Since system poles do not depend on the steady-state. no such correction is needed for poles. It should be mentioned here that as in all small-signal analyses, the accuracy of the model drops as the operating point moves away from the assumed steady-state operating point.

\section{TRANSFFr FIN TIONSOF OPIN-Lox)P SYSTI:M}

In order to derive the small-signal transfer functions around the time-varying steady-state defined by $X=\left(\begin{array}{lll}X_{1} & X_{2} & X_{3}\end{array}\right)^{T}$ and duty ratio $D$ ) at time $1-()$. a small perturbation $\tilde{d}$ of duty ratio is introduced at time $t=0$ that causes corresponding changes of state variables given by $\bar{x}_{i}$ where $i=1,2$, or 3 . I3y substituting the instantaneous values of states and the duty ratio as $x_{i}=x_{i}+x_{j}$ and $d=D+\dot{d}$. the state equation $(6)$ can be rewritten as.

$$
\dot{x}+\dot{\tilde{x}}=\left[A_{1} \cdot(D+\tilde{d})+A_{2} \cdot(1-D-\dot{d})\right] \cdot(X+\tilde{x})
$$

By neglecting the product of small variations. the steady state values can be separated as.

$\dot{X}=\left[A_{1} \cdot D+A_{2} \cdot(1-D)\right] \cdot X=A_{1} \cdot X$

where $A_{0}=A_{1} \cdot D+A_{2} \cdot(1-D)$ is the initial state matrix at $t=0$ and it is given by (7) with all $d$ terms replaced by $D$. As described in section II.B.2. in order to satisfy this equation, a time-varying steady-state has to be considered. The only difference it makes compared to a de steady state is the frequency shift of $\omega_{1}$ applied to zeros.

Similarly, the perturbed values of states are separated from (16) as.

$\hat{x}=A_{0} \cdot \bar{x}+\left[A_{1}-A_{2}\right] \cdot X \cdot \hat{d}$

By taking Laplace transform of (17), perturbed values of states are given by.

$\tilde{r}=\left(s I-A_{0}\right)^{-1} \cdot\left[A_{1}-A_{2}\right] \cdot X \cdot \bar{d}$

where $s$ is the complex frequency, $I$ is a $3 \times 3$ unit matrix. Tho state transfer functions with respect to duty ratio can then be derived as,

$$
\begin{aligned}
{\left[G_{1}(s) G_{2}(s) G_{3}(s)\right]^{T} } & =\left[\frac{\tilde{x}_{1}(s)}{\tilde{d}(s)} \frac{\tilde{x}_{2}(s)}{\tilde{d}(s)} \frac{x_{3}(s)}{d(s)}\right]^{T} \\
& =\left[s \cdot 1-A_{0}\right]^{-1}\left(A 1-A_{2}\right) X
\end{aligned}
$$

If the state transler functions are written as ratios of numerator and denominator functions.

$\left[G_{1}(s) G_{2}(s) G_{3}(s)\right]^{T}=\left[\frac{N_{1}(s)}{P(s)} \frac{N_{2}(s)}{P(s)} \frac{N_{3}(s)}{P(s)}\right]^{T}$

they can be derived using (19) as,

$$
\begin{aligned}
& P(s)=s^{3}+\left[1:\left(R C_{1}\right)\right]_{-}^{2}+\frac{1}{L}\left[( 1 - D ) ^ { 2 } \left(C_{1}+1 C_{t}{ }_{-}{ }^{-}+\left[\frac{1}{I C_{1 t} C_{t} R}\right](21)\right.\right. \\
& X_{1}(s)=-\frac{1}{\left(L C_{11} C_{1} R\right)}\left[\left(C_{1} R X_{3}\right) s+\left(X_{3}+(1-D) R X_{2}\right)\right] \\
& N_{2}(s)=\frac{s}{(L C, R)}\left[\left(C, R I_{3}\right) s+\left(X_{3}+(1-D) R X_{2}\right)\right] \\
& X_{i}(s)=-\frac{1}{\left(L C_{n} C_{1}\right)}\left[\left(I, C_{n}, X_{2}\right) s^{2}-\left((1-D) C_{n}, X_{3}\right) s+X_{2}\right]
\end{aligned}
$$

Since $x_{3}$ is the output variable, the output transter function is given by, $G_{3}(s)=\frac{N_{3}(s)}{P(s)}=\frac{-X_{2}}{C_{t}} \cdot \frac{\left(s-z_{1}\right)\left(s-z_{2}\right)}{\left(s-p_{1}\right)\left(s-p_{2}\right)\left(s-p_{3}\right)}$

where $p_{1}, p_{2}$ and $p_{3}$ are the system poles and $z_{1}$ and $z_{2}$ are the system zeros.

\section{A. Exact Values of Poles in (open-Loop Sistem}

The characteristic equation of the open-loop system is given by $P(s)=0$. It can also be written as.

$$
\left(L C_{1 H} C^{\prime}, R\right) s^{3}+\left(L C_{1 t}\right) s^{2}+R\left[C_{11}(1-D)^{2}+C_{f}\right] s+1=0
$$

According to (26), system open-loop poles do not depend on the steady-state values of state variables. It depends only on the steady-state value of the duty-ratio and the circuit parameters. Analytical expressions for the three poles of the open-loop system can be derived by using a symbolic mathematical routine such as 'solve' of symbolic mathematics toolbox of Matlab. However, the resulting expressions are too complex for any practical analysis and therelore are not given here. Instead. approximate pole positions can be derived by considering the low frequency and high frequency behaviour of the system separately.

B. Approximate Value of Lon-Frequency Pole:

Due to the complexity of analytical expressions of exact values of poles, two possible approximations are proposed in this section.

1. Approximation 1:

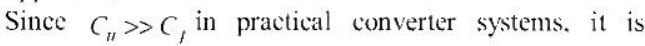
reasonable to neglect $C_{f}$ in (26) if the duty ratio satisties the condition, $C_{n}(1-D)^{2} \gg C_{1}$, i.e.

$D \ll 1-\sqrt{\frac{C_{f}}{C_{n}}}$

The resulting characteristic equation for low frequency behaviour can be expressed as.

$\left(L C_{u}\right) s^{2}+R C_{u}(1-D)^{2} s+1=0$ 
The two poles corresponding to this equation can be written as.

$$
\begin{aligned}
& p_{1}^{*}=\frac{-R(1-D)^{2}}{2 L}\left[1-\sqrt{1-\frac{4 L}{C_{t \prime}\left(R(1-D)^{2}\right)^{2}}}\right] \\
& p_{4}^{*}=\frac{-R(1-D)^{2}}{2 L}\left[1+\sqrt{1-\frac{4 L}{C_{u}\left(R(1-D)^{2}\right)^{2}}}\right]
\end{aligned}
$$

It can be found that the term within the square root sign is positive and the two poles are real as long as the duty ratio is below a certain limit value given by,

$D_{11}=1-\sqrt{\frac{2}{R} \sqrt{\frac{l}{C_{11}}}}$

However, considering the practical values for the eircuit parameters it can be seen that $D$, is very close to unity. Since such high duty ratios are impractical and also violate the condition in (27), it is reasonable to assume that the two poles are real and negative in the practical range of duty ratio. Out ol the two poles on the real axis of Left Half Plane (LHP) of complex s-plane, $p_{1}^{*}$ has a small negative value and $p_{4}^{*}$ has a large negative value. i.e. $\left|p_{1}^{*}\right| \ll\left|p_{4}^{*}\right|$. Since $p_{4}^{*}$ is out of the focused low frequency range. it should be ignored and only $P_{1}^{*}$ should be taken as the possible approximate value of the low frequency pole of the system.

\section{Appreximation 2 :}

If necessary, (29) can be expressed in terms of initial state values by noting in $(9)$ and $(10)$ that $R(1-D)^{2}=x_{1}, X_{2}$. The equation can be further simplified by approximating the term within square root sign as $\sqrt{1-x}=1-\frac{x}{2}$ since $\mathrm{x}<1$ when $D<D_{\imath}$.

$p_{1}^{n x}=\frac{-X_{1}}{2 L X_{2}}\left[1-\left(1-\frac{1}{2}\left\{\frac{A L}{C_{11}\left(X_{1} X_{2}\right)^{2}}\right\}\right)\right]=\frac{-X_{2}}{\left(C_{11} X_{1}\right)}=-\omega_{1}$

This result can also be verified from the differential equation $\dot{x}_{1}=\left(-1 / c_{u}\right) x_{2}$ that appears in (6) and (7). This equation when converted to s-domain can be written as $x_{1}=\left(-x_{2} / C_{u}\right) \Rightarrow\left(s+x_{2} /\left(C_{u} x_{1}\right)\right)=0$. Thus, the low-frequency pole at any time is given by $-x_{2} /\left(C_{t} x_{1}\right)$. The second possible approximate value of the pole $p_{1}^{* *}$ is therefore equal to $-\omega_{1}$ at the steady-state.

C.. Approximate Values of High-Frequency Poles

Next, the characteristic equation describing the high frequency behaviour of the system ean be derived by substituting $C_{u} \rightarrow \infty$ in (26) as,
$s^{2}+\left[1,\left(R C_{1},\right)\right] s+\left[(1-D)^{2}\left(L C^{\prime},\right)\right]=0$

The two poles resulting from the above equations are given by. $p_{2}=-1\left(2 R C_{1}\right)+i \sqrt{(1-I))^{2}\left(L C_{1}\right)-1\left(2 R C_{1}\right)^{2}}$

$p_{3}^{*}=-1\left(2 R C_{1}\right)-i \sqrt{(1-D)^{2}\left(L C_{1}\right)-1 \cdot\left(2 R C_{1}\right)^{2}}$

The two poles are complex conjugates with a large negative real part as long as the duty ratio is below another limit given by.

$D_{12}=1-\frac{1}{2 R} \sqrt{\frac{L}{C_{t}}}$

Once again. the limiting value is very close to unity and is therefore out of the practical range.

\section{Zeres of (Output Tiansfer Function}

By substituting (9) and (10) in (24). $N_{3}(s)$ can be expressed for running condition as.

$N_{3}(s)=-\frac{X_{2}}{\left(C_{f}\right)}\left[s^{2}-\left(\frac{(1-D)^{2} R}{L}\right) s+\left(\frac{1}{L C_{H \prime}}\right)\right]$

The roots of (37) ean then be derived as,

$z_{i k l}=\frac{R(1-D)^{2}}{2 L}\left[1-\sqrt{1-\frac{4 L}{C_{u}\left(R(1-D)^{2}\right)^{2}}}\right]$

$z_{d L_{2}}=\frac{R(1-D)^{2}}{2 L}\left[1+\sqrt{1-\frac{4 L}{C_{u}\left(R(1-D)^{2}\right)^{2}}}\right]$

Note here that $z_{k+1}$ and $z_{k, 2}$ are the zeros of the output transfer function if a de steady-state is assumed. By comparing $(38)$ and (39) with (29) and (30) respectively, it can be seen that $z_{d l_{1}}=-p_{1}^{*}$ and $z_{d k_{2}}=-p_{4}^{*}$. That means. both $z_{d k 1}$ and $z_{d k 2}$ lie on the real axis ol Right Half Plane (RHP) of the complex s-plane so that $z_{d \cdot 1} \ll z_{d \cdot 2}$.

By considering the frequency shift due to the time-varying steady-state, the zeros of the output transfer lunction can be calculated as.

$z_{1}=z_{(k)}-\left(q_{1}\right)=-p_{1}-\left(q_{1}=\omega_{1}-\left(q_{1}=0\right.\right.$

$\left.z_{2}=z_{k_{2} 2}-\omega_{1}\right)\left(\frac{X_{1}}{L X_{2}}-\omega_{1}\right)-\omega_{1}=\frac{X_{1}}{L X_{2}}-2 \omega_{1}$

Hence, for all practical purposes, the zero $z_{1}$ can be treated as at the origin. The remaining zero $z_{2}$ lies on the real positive axis of s-plane far away from the origin. Consequently, all the well known problems due to RHP zero of a classical boost converter. such as undershooting and limitations on controller gain. $[13,14]$ are expected to remain the same when supplied with a bank of ultracapacitors. 
E. Comparison with the Comerter Suppled Br a Constant Foltage Source

Conventionally, batteries with relatively constant voltage wero used to supplement energy in applications where ultracapacitor banks are currently being used. Therefore. it is important to make a comparison of pole zero positions due to these two types of energy sources. The appearance of one RHP 7ero and the corresponding undershooting in the step response of the classical boost converter supplied by a constant voltage source are well documented [14]. The same information can be derived here by making $C_{u} \rightarrow \infty$ in the pole zero expressions derived for the ultracapacitor based converter.

As given by (29) and (32). both the approximate values of dominant pole $p_{1}^{*}$ and $p_{1}^{* *}$ approaches zero as $C_{u} \rightarrow \infty$. Therefore $p_{1}$ and $z_{1}$ eancel with each other under this condition and disappears from the transfer function. The approximate values of high-frequency poles $p_{2}^{*}$ and $p_{3}^{*}$ do not change with $C_{n}$ as the assumption $C_{n}^{\prime} \rightarrow \infty$ was made during the derivation of their values. Therefore, $p_{2}$ and $p_{3}$ ean be identified as the two poles of the system supplied by a constant voltage source, such as a battery. Since the steady-state frepuency $\omega_{1} \rightarrow 0$ as $C_{u} \rightarrow \infty$, the zero $z_{2}$ reaches the maximum possible value when $C_{u} \rightarrow \infty$ is substituted in (41) as,

$z_{h}=\frac{X_{1}}{L X_{2}}=\frac{R(I-I))^{2}}{L}$

where $z_{b}$ is the single zero of the constant voltage source supplied classical boost converter. Thus, the small-signal transfer function of the constant voltage source supplied system can be derived using (25) as.

$$
G_{b}(s)=\frac{-X_{2}}{C_{1}} \cdot \frac{\left(s-z_{b}\right)}{\left(s-p_{2}\right)\left(s-p_{3}\right)}
$$

Moreover, the zero of the ultracapacitor supplied boost converter. $z_{2}$, can be expressed in terms of $z_{h}$ as.

$z_{2}=z_{h}-2 \omega_{1}$

IV. II.LLSTRATIVL EXAMPLE:

To demonstrate the control characteristics of the ultra-capacitor supplied classical boost converter, a practical system with following parameters were considered. Capacitance per cell = I00F. Number of Cells in series $=44 . \therefore C_{u}=100 / 44=2.273 \mathrm{~F}$. Rated Load Voltage $I_{r}=100 \mathrm{~V}$. Minimum Duty Ratio $D_{m m}=0 . \mathrm{I}$, Maximum Duty Ratio $D_{m, n}=0.8$. Converter Inductance $L=4.2 \mathrm{mH}$, Converter Filter Capacitance $C_{1}=79 \mu \mathrm{F}$.

\section{A. Trevification of the Time-l'aring Steadr-State}

Considering two initial values of state variables as $X_{1}=50 \mathrm{I}^{\circ} . X_{3}=100 \mathrm{I}^{\circ}$ and the load resistance as $10 \Omega$, the remaining state variable, duty ratio and the steady-state frequency can be calculated using (12)-(14) as
$I_{2}=2(0, A, I)=0.5, \omega_{1}=0.176 \mathrm{rad} \mathrm{s}$. Figure 2 illustrates how the three state variables vary in the steady-state if the duty ratio is kept constant at $D=(1.5$. The simulations were performed using Matlab/Simulink. By the comparison of the simulation results with values calculated using the assumed mathematical model in (11). the high accuracy of the model given by (II) and the variables calculated using (12)-(14) can be verified.

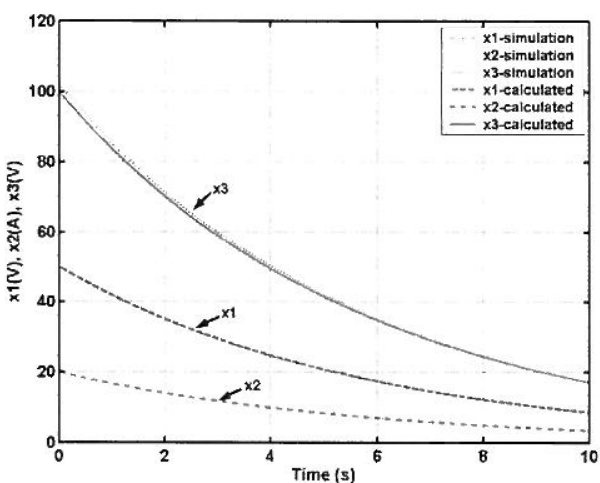

Fig. 2 Simulation results and the calculated values usmg the assumed mathematical model for the variations of the state variables with a constant duty ratto

\section{B. Variations of Poles and Zero with Dutr Ratio}

By considering a load resistance of $22.22 \Omega$ corresponding to a rated load eurrent of $4.5 \mathrm{~A}$, the critical values of duty ratios can be determined using (22) and (26) as, $D_{1}=0.938$ and $D_{t 2}=0.836$ respectively. Since $D_{m a t}<D_{21}$. the pole $p_{l}$ will be real and negative throughout the operating range. Similarly. $D_{m a 1}<D_{2}$ means that the two poles $p_{2}$ and $p_{3}$ will be complex conjugates with negative real parts for the entire operating range. In order to study the effects of changing duty ratio on the system poles and zeros and also to study the sizes of crrors in their approximate values, the duty ratio is changed in the operating range from 0.1 to 0.8 . Figure 3 (a) illustrates how the exact values of poles $p_{1}, p_{2}$ and $p_{3}$, calculated by solving (26). and the zero $z_{2}$ vary with the duty ratio. This figure clearly shows that the zero has the highest frequency during most of the range of duty ratio. The zero moves closer to origin as the duty ratio is increased. The pair of high frequency poles $p_{2}$ and $p_{3}$ does not appear to move closer to origin as the duty ratio is increased. Furthermore, compared to the pair of high frequency poles, the magnitude of dominant pole in the entire range of duty ratio is seen to be nearly zero.

Through the study made by changing the duty ratio, it is also possible to estimate the accuracy of approximate values of poles and zeros. The percentage errors of two approximate values of the dominant pole $p_{1}^{*}$ and $p_{1}^{* *}$, the zero $z_{1}$, the real 
and imaginary parts of approximate value $p_{2}^{*}$ are plotted against the duty ratio in Fig. 3(b). As observed from this figure. the errors of all the values except that of $z$ are below $0.1 \%$ during the full range of duty ratio. In the case of $z_{1}$ the error is up to $0.2 \%$. As the derived equations suggested. all the errors increase with increasing duty ratio. Interestingly. the error of the second approximation of the dominant pole $p_{1}^{* *}$ is less than that of $p_{1}^{*}$ and is nearly zero up to a duty ratio of about 0.7 . Therefore, the dominant pole can be approximated with high accuracy by $p_{1}^{*}=-c_{1}$. Since the error in approximation of both zeros is the same. the error of $z_{2}$ is not shown in the ligure. In briel: all the approximated values are highly accurate and suitable for the purpose of designing controllers. Through several case studies. it was confirmed that the pole-zero variations and the accuracy of approximation of poles are typical to most practical converter systems.

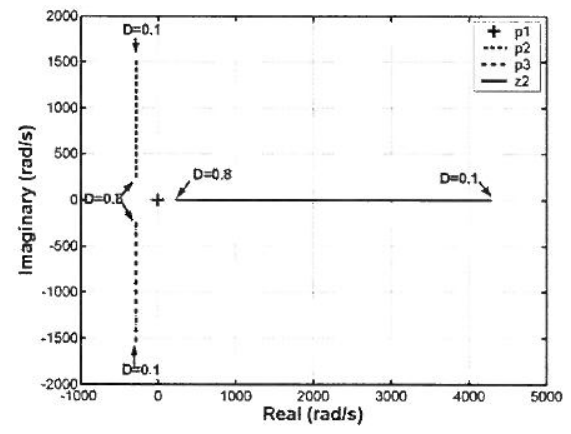

(a)

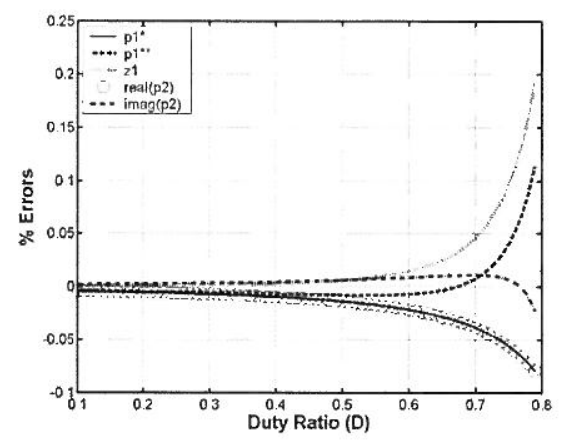

(b)

Fig. 3. Elfects of Varying Duty Ratio (a) all poles and sero (b) percentage errors of approximate values.

V. CONCLUSIONS

Due to the increasing industrial applications of ultracapacitors, it is imperative to understand the characteristics of power electronic converters when supplied by banks of ultracapacitors. The variable-voltage at the terminals make the control characteristics of such systems to be significantly diflerent from those supplied by constant voltage sources such as batteries. Based on a time-varying steady-state, system transler functions, their poles and zeros are derived in this paper for a classical boost converter supplying a resistive load. In order to reduce the mathematical complexity. simple equations have been derived to yield highly accurate approximate values for poles and zeros. By deriving the corresponding transter function for a constant voltage source supplied converter. it clearly demonstrates that the main difference between them is the presence of a low-frequency pole on LHP and a zero at the origin in the ultracapacitorsupplied system transter limction. Through the approximate values derived, it shows that the additional pole is due to the capacitive nature of the source. Hence the additional pole is expected to be present in general in the transfer functions derived for any power electronic converter supplied by a bank of ultracapacitors.

\section{RFFERENCES}


fundememtals and ted holengis al applications. New York: Kluwer Academic/ Plenum Press, 1999.

[2] A Burke. "Litracapacitors why. how. and where is the technology.".Journal of Perwer Serures, vol. 91. pp. 37-50. 2000)

[3] R. Kotz and M Carlen. "Principles and applications of electrochemical capacitors." Electrochimica te ta. vol. $45 . \mathrm{pp}$ electrohemical con

[4] M. Corley. J. Locker. S. Dutton, and R. Spee. "L'Itracapaciturbased ride-through system for adjustable speed drives," 30th Annual IEEE Power Electronic Specialists conference. 1949.

[5] A. Schneuwly, M Bärschi, V. Hermann. (i. Sartorelli, R. (jallay. and R. Kotz. "BoOSTCAP Double-Laver (apacitors for Peak Power Automotive Applications," Second International Advanced Automative Battery (onference. 200)?

[6] IS. I.ai. S I.evy, and M. F. Rose. "High energy density doublelayer capacitors for energy storage applications." JEEF. Acruspecte and Eletronic Sistem Magazime', vol. 7. pp. 14-19. 1992

[7] J. I.. Duran-Giomez. P. N. Fnjeti, and A. von Jouanne, "An approach to achieve ride-through of an adjustable-speed drive with flyback converter modules powered by suner capacitors." IEEE Transactions on Indistry . Applicatiom, vol. 38. pp. 514-522, 20(1)2

[8] M. P. Kaàzmierkowski. R. Krishnan. and F. Blaabjerg. Control in poner destronics selceted problems. Amsterdam. Beston Academic Press. 2002

[9] R. W. Frickson and D. Maksimeriàc, Fundementals of porues. cketromis. 2nd ed. Chapman \& Hall. New York, 1997

[10] F. Belhachemi. S. Rael, and B. Davat."A physical based model of power electric double-layer supereapacitors"."Industry
Applications Conference. vol .5.2000. pp. 3(1)69-3076.

[11] S. Buller, E. Karden, D. Kolk. and R. W. De Doncker. "Moxielin! the dynamic behavior of supercapacitors using impedance spectroscopy".IEEE Trams. on Industry Applications. vol. 38. 2002. pp. 1622-1626.

[12] R. D. Middlebrook, "Small-signal modeling of pulse-width modulated switched-mode power converters". Proc of the IEEE. vol. 76. No 4. Apr 1988, pp. 34,3-354.

[13] K. J. Astrom. "Limitations on control system perlormance"." Europeetur Journal of Control". vol. 6. No. 1, 20)(0).

[14] K Viswanathan. R. Oruganti. and D. Srimivasan. "A novel tri-state boost converter with fast dynamics." IEEE Transat toms on Powe'r Elestrents s, vol. 17, pp. $677-683,2002$ 